\title{
Natural Produce Classification Using Computer Vision Based on Statistical Color Features and Derivative of Radius Function
}

\author{
Anton Satria Prabuwono ${ }^{1,2, a^{*}}$, Joko Siswantoro ${ }^{1,3, b}$, Azizi Abdullah ${ }^{1, c}$ \\ ${ }^{1}$ Faculty of Information Science and Technology, Universiti Kebangsaan Malaysia, \\ 43600 UKM Bangi, Selangor D. E., Malaysia \\ ${ }^{2}$ Faculty of Computing and Information Technology, King Abdulaziz University, \\ Rabigh 21911, Saudi Arabia \\ ${ }^{3}$ Faculty of Engineering, Universitas Surabaya, Jl. Kali Rungkut, Surabaya, 60293, Indonesia \\ aantonsatria@eu4m.eu, bjoko_siswantoro@ubaya.ac.id, cazizi@ftsm.ukm.my
}

\begin{abstract}
Keywords: Natural produce classification, Computer vision, Statistical color features, Derivative of radius function, $k$-nearest neighbors, Artificial neural network.
\end{abstract}

\begin{abstract}
In agriculture industry, natural produce classification is used in sorting, grading, measuring, and pricing. Currently, a lot of methods have been developed using computer vision to replace human expert in natural produce classification. However, some of the method used long features descriptor and complex classifier to obtain high classification rate. This paper proposes natural produce classification method using computer vision based on simple statistical color features and derivative of radius function. The $k$-nearest neighbors $(k$-NN) and artificial neural network (ANN) were used to classify the produce based on the extracted features. Preliminary experiment results show that the proposed method achieved best result with average classification accuracy of $99.875 \%$ using ANN classifier with nine nodes in hidden layer.
\end{abstract}

\section{Introduction}

Natural produce classification aims to classify the produce into one of classes. In agriculture industry, natural produce classification is used in sorting [1], grading [2], measuring [3], and pricing [4]. Usually, natural produce classification is performed manually by human expert. However, manual classification is inaccurate and difficult to standardize [2]. Currently, a lot of methods have been developed using computer vision to replace human expert in natural produce classification [1, 2, 4-9]. Generally, an image of produce is acquired and then processed to extract its features such as color, shape, and texture. The produce is then classified using a classifier based on the extracted features.

VeggieVision [4] is the first produce recognition system. The system was developed to recognize a produce in supermarket and grocery stores during weighing in order to identify the price of produce. VeggiVision used color, texture, size, and density features; and nearest neighbors classifier. The best accuracy of $95 \%$ was reported, however this result was achieved in the top four choices. Kilıç, et al. [2] proposed a classification system for quality evaluation of beans using computer vision. They used statistical color features as input for artificial neural network (ANN) classifier. The correct classification rate of $90.6 \%$ was achieved. Roomi, et al. [1] proposed mangoes classification method object contour modeling and region base descriptor. Bayes classifier was used and obtained classification accuracy of 90.91\%. Woo Chaw and Mirisaee [9] proposed a method for fruit recognition based on color and shape features. The proposed method used $k$-nearest neighbors $(k-\mathrm{NN})$ classifier and was accurate up to $90 \%$.

Recently, some researcher used a combination of very long features descriptor for produce recognition, such as Unser's descriptors, color coherence vectors, border/interior, global color histogram, appearance descriptors, color autocorrelogram, local activity spectrum, quantized compound change histogram, and edge orientation autocorrelogram $[5,6,8]$. Furthermore, they also used a complex classification method to obtain classification accuracy more than $97 \%$, namely fusion of support vector machine (SVM) with radial basis function (RBF) kernel [5], bagging 